\title{
Clinical Treatment of Elderly Patients with Endometrial Cancer
}

\author{
Taiki Kikuchi, Yasunori Hashiguchi*, Makoto Yamauchi, Mari Kasai, Takeshi Fukuda, Tomoyuki Ichimura, \\ Tomoyo Yasui and Toshiyuki Sumi \\ Department of Obstetrics and Gynecology, Osaka City University Graduate School of Medicine, Japan
}

Submission: January 27, 2021; Published: February 02, 2021

*Corresponding author: Yasunori Hashiguchi, Department of Obstetrics and Gynecology, Osaka City University Graduate School of Medicine, Japan

\begin{abstract}
Background: There is some reports investigating clinical management or survival of elderly patients with endometrial cancer in large case series. However, the standard treatment remains controversial in elderly patients with endometrial cancer. In this study, we investigated clinical treatment and outcomes of elderly patients with endometrial cancer.

Methods: This retrospective study was approved by the Institutional Review Board of the institution. Between January 2014 and December 2019, clinical data were collected in patients with endometrial cancer. The patients were divided two group: elderly patients (age: 75 years or older than 75) and younger patients (age: younger than 75). We examined clinical treatment and outcomes of elderly patients.

Results: A total of 298 patients were treated for endometrial cancer. The patients were divided two group: 43 elderly patients and 255 younger patients. Subsequently 52 patients (24.2\%) relapsed and the mean period of follow-up was 45 months (1-95). Endometrioid tumors were more frequently diagnosed in younger group $(\mathrm{p}=0.002)$, while histological grade 3 cancer and myometrial invasion depth $>1 / 2$ were more frequently diagnosed in elderly group $(\mathrm{p}=0.003 ; \mathrm{p}=0.002)$. There were no significant differences in terms of clinical stages, lymph node metastases, or distant metastases. Elderly patients received less pelvic lymphadenectomy than younger patients $(\mathrm{p}=0.0004)$. There were no significant differences in terms of hysterectomy, or para-aortic lymphadenectomy. In the Elderly patients, rates of optimal oncologic therapy according to the treatment principle of the institution was slightly lower compared to younger patients (86.0\% vs. 93.7\%), however the difference was not statistically significant. The elderly patients had significantly frequent relapse of disease (32.6\%) more than the younger patients $(10.6 \%)$ $(\mathrm{p}=0.0005)$.
\end{abstract}

Conclusion: The elderly patients with endometrial cancer had significant poorer prognosis than the younger patients, and reasons for this include both high risk tumor biology and less aggressive operation.

Keywords: Endometrial cancer; Elderly patients; Clinical treatment

\section{Introduction}

Endometrial cancer is common cancer, and its incidence is increasing globally [1-8]. The number of elderly patients with endometrial cancer has gradually increased as well [1,2]. The standard therapy for endometrial cancer includes comprehensive surgical operation and adjuvant chemotherapy. Generally, elderly patients are less likely to be offered operative therapy, lymph node dissection, and adjuvant chemotherapy [2-6].

Previous reports showed that advanced age is a predictor of poor prognosis in patients with endometrial cancer [2]. Although advancing age is associated with an increasing tendency toward high-grade endometrioid carcinoma, the histological characteristics do not appear to be able to entirely indicate the unfavorable prognosis of elderly patients with endometrial cancer $[2,6]$. In this study, we investigated clinical treatment and outcomes of elderly patients with endometrial cancer.

\section{Methods}

This retrospective study was approved by the Institutional Review Board. Available clinical records from January 2014 to December 2019 were reviewed, and the patients were divided two group: elderly patients (age: 75 years or older than 75 ) and younger patients (age: younger than 75 ). We examined clinical treatment and outcomes of elderly patients. 
Clinical treatment of endometrial cancer in our institution was performed by gynecologic oncologists and diagnoses were established by curettage. Patients were classified according to the FIGO surgical staging system (2008). Pathological diagnoses were confirmed according to the World Health Organization criteria. In operable cases, patients received hysterectomy plus bilateral salpingo-oophorectomy. Peritoneal fluid was obtained for cytological examination, and pelvic lymphadenectomy was performed. Para-aortic lymphadenectomy was performed in patients with high or intermediate risk disease. In patients without lymphadenectomy, lymph nodes of $>1 \mathrm{~cm}$ were detected using radiologic imaging test and were considered positive lymph nodes. Adjuvant chemotherapy was performed for patients with high or intermediate risk disease. Patient treatments were followed with gynecologic examinations including ultrasonography and cytological examination of vaginal cut edges, and laboratory tests including tumor markers. Further imaging tests were performed for patients with clinical symptoms or elevated tumor marker levels. Recurrent disease was diagnosed using imaging methods or biopsy.

\section{Statistical Analysis}

Statistical analysis between clinical groups was performed using Fisher's exact probability test. In the analyses of disease relapse, survivals were calculated using the Kaplan-Meier method. Cox regression analyses were performed to identify variables associated with relapse-free survival such as clinical data and pathological data. $\mathrm{P}<0.05$ was considered statistically significant.

\section{Results}

\section{Patient characteristics}

Patient characteristics are shown in Table 1. A total of 298 patients (stage I, 218; II, 21; III, 39; IV, 20) were administrated. The patients were divided two group: 43 elderly patients and 255 younger patients. The median age was 57 years (29-92), and histology types included 266 endometrioid carcinomas, 14 serous carcinomas, 13 carcinosarcomas, and five clear cell carcinomas. Histological grades were defined as grades 1, 2, and 3 in 174, 57, and 67 cases, respectively. Surgery was performed in 287 cases (96.3\%). Between 11 inoperable cases (3.7\%), chemotherapy was performed in eight cases $(2.7 \%)$, and radiation therapy was performed in three cases (1.0\%). Surgery included hysterectomy plus bilateral salpingo-oophorectomy in 287 patients (96.3\%) and additional lymphadenectomy was performed in 253 patients (84.9\%). Para-aortic lymphadenectomy was performed in 78 patients $(26.2 \%)$. Adjuvant chemotherapy was performed for 141 patients $(47.3 \%)$ with high or intermediate risk disease, and included paclitaxel and carboplatin therapy (TC) in all patients. TC therapy was performed for eight inoperable cases, and 41 patients $(13.8 \%)$ had relapse of disease. The mean period of follow-up was 39 months (1-83).

Table 1: Patient characteristics.

\begin{tabular}{|c|c|c|c|}
\hline & Population $(n=298)$ & Elderly patients $(n=43)$ & Younger patients $(n=255)$ \\
\hline Age (years) & $57(29-92)$ & $81(75-92)$ & $54(29-74)$ \\
\hline \multicolumn{4}{|l|}{ Histology } \\
\hline endometrioid & $266(89.3 \%)$ & $32(74.4 \%)$ & $234(91.8 \%)$ \\
\hline serous & $14(4.7 \%)$ & $6(14.0 \%)$ & $8(3.1 \%)$ \\
\hline carcinosarcoma & $13(4.4 \%)$ & $4(9.3 \%)$ & $9(3.5 \%)$ \\
\hline clear & $5(1.7 \%)$ & $1(2.3 \%)$ & $4(1.6 \%)$ \\
\hline \multicolumn{4}{|l|}{ Stage } \\
\hline 1 & $218(73.2 \%)$ & $26(60.5 \%)$ & $192(75.3 \%)$ \\
\hline 2 & $21(7.0 \%)$ & $6(14.0 \%)$ & $15(5.9 \%)$ \\
\hline 3 & $39(13.1 \%)$ & $7(16.3 \%)$ & $32(12.5 \%)$ \\
\hline 4 & $20(6.7 \%)$ & $4(9.3 \%)$ & $16(6.3 \%)$ \\
\hline \multicolumn{4}{|l|}{ Histological grade } \\
\hline 1 & $174(58.4 \%)$ & $15(34.9 \%)$ & $159(62.4 \%)$ \\
\hline 2 & $57(19.1 \%)$ & $10(23.3 \%)$ & $47(18.4 \%)$ \\
\hline 3 & $67(22.5 \%)$ & $18(41.9 \%)$ & $49(19.2 \%)$ \\
\hline \multicolumn{4}{|l|}{ Performance status } \\
\hline $0-1$ & $283(95.0 \%)$ & $30(69.8 \%)$ & $253(99.2 \%)$ \\
\hline$\geq 2$ & $15(5.0 \%)$ & $13(30.2 \%)$ & $2(0.8 \%)$ \\
\hline \multicolumn{4}{|l|}{ Comorbidity } \\
\hline Diabetes & $41(13.8 \%)$ & $9(20.9 \%)$ & $32(12.5 \%)$ \\
\hline
\end{tabular}




\section{Journal of Gynecology and Women's Health}

\begin{tabular}{|c|c|c|c|}
\hline Heart disease & $12(4.0 \%)$ & $3(7.0 \%)$ & $9(3.5 \%)$ \\
\hline Cerebral infarction & $7(2.3 \%)$ & $3(7.0 \%)$ & $4(1.6 \%)$ \\
\hline Psychiatric disorder & $17(5.7 \%)$ & $2(4.7 \%)$ & $15(5.9 \%)$ \\
\hline other & $35(11.7 \%)$ & $8(18.6 \%)$ & $27(10.6 \%)$ \\
\hline
\end{tabular}

\section{Clinical and treatment-related characteristics}

Clinical characteristics are provided in Table 2. Endometrioid tumors were more frequently diagnosed in younger group $(80.8 \%$ vs. $73.2 \%)(p=0.002)$, while histological grade 3 -endometrial cancer was more frequently diagnosed in elderly group ( $41.9 \%$ vs.
$14.0 \%$ ) ( $\mathrm{p}=0.003)$. Tumor with myometrial invasion depth $>1 / 2$ was also more frequently diagnosed in elderly patients $(65.1 \%$ vs. $39.2 \%)(p=0.002)$. There were no significant differences between both groups in terms of clinical stages, lymph node metastases, or distant metastases.

Table 2: Clinical characteristics.

\begin{tabular}{|c|c|c|c|}
\hline & Elderly patients $(n=43)$ & Younger patients $(n=255)$ & $P$ value \\
\hline \multicolumn{4}{|l|}{ Stage } \\
\hline $\mathrm{I}+\mathrm{II}$ & $32(74.4 \%)$ & $207(81.2 \%)$ & NS \\
\hline III+IV & $11(15.6 \%)$ & $48(18.8 \%)$ & \\
\hline \multicolumn{4}{|c|}{ Histological grade } \\
\hline $1+2$ & $25(58.1 \%)$ & $206(60.5 \%)$ & 0.003 \\
\hline 3 & $18(41.9 \%)$ & $49(14.0 \%)$ & \\
\hline \multicolumn{4}{|l|}{ Histology } \\
\hline endometrioid & $32(73.2 \%)$ & $234(80.8 \%)$ & 0.002 \\
\hline other & $11(7.0 \%)$ & $21(19.2 \%)$ & \\
\hline \multicolumn{4}{|c|}{ Lymph node metastasis } \\
\hline negative & $39(90.7 \%)$ & $227(89.0 \%)$ & NS \\
\hline positive & $4(9.3 \%)$ & $28(11.0 \%)$ & \\
\hline \multicolumn{4}{|c|}{ Distant metastasis } \\
\hline negative & $39(90.7 \%)$ & $239(93.7 \%)$ & NS \\
\hline positive & $4(9.3 \%)$ & $16(6.3 \%)$ & \\
\hline \multicolumn{4}{|c|}{ Myometrial invasion } \\
\hline$<1 / 2$ & $15(34.9 \%)$ & $155(60.8 \%)$ & 0.002 \\
\hline$\geq 1 / 2$ & $28(65.1 \%)$ & $100(39.2 \%)$ & \\
\hline
\end{tabular}

Treatment-related characteristics are provided in Table 3. Elderly patients received less pelvic lymphadenectomy $(65.1 \%$ vs. $88.2 \%)(p=0.0004)$ than younger patients. There were no significant differences between both groups in terms of hysterectomy, or para-aortic lymphadenectomy. In the Elderly patients, rates of optimal oncologic therapy according to the treatment principle of the institution was slightly lower compared to younger patients ( $86.0 \%$ vs. $93.7 \%$ ), however the difference was not statistically significant. The elderly patients had significantly frequent relapse of disease $(32.6 \%)$ more than the younger patients $(10.6 \%)(\mathrm{p}=0.0005)$.

\section{Discussion}

Endometrial cancer is the common gynecologic malignancy in women, and its incidence is increasing globally [1-8]. The average age at diagnosis is approximately 68 years [2]. The number of elderly patients with endometrial cancer has gradually increased in Japan as well. Most patients present with early-stage disease, however, about $25 \%$ of patients present with stage III or stage IV and will require comprehensive treatment including adjuvant therapy [1-8]. The standard therapy for endometrial cancer remains controversial, but it mainly includes comprehensive 
surgical staging operation and adjuvant chemotherapy for patients with high or intermediate risk disease [2-6]. Generally, elderly patients with endometrial cancer are less likely to be offered operative therapy, lymph node dissection, and adjuvant chemotherapy [2].

Table 3: Treatment-related characteristics.

\begin{tabular}{|c|c|c|c|}
\hline & Elderly patients (n=43) & Younger patients (n=255) & P value \\
\hline Hysterectomy & & & $248(97.3 \%)$ \\
\hline Yes & $39(90.7 \%)$ & $7(2.7 \%)$ & NS \\
\hline No & $4(9.3 \%)$ & & $225(88.2 \%)$ \\
\hline Pelvic lymphadenectomy & & $30(11.8 \%)$ & 0.0004 \\
\hline Yes & $28(65.1 \%)$ & & \\
\hline No & $15(34.9 \%)$ & $183(71.8 \%)$ & NS \\
\hline Para-aortic lymphadenectomy & $6(14.0 \%)$ & & \\
\hline Yes & $37(86.0 \%)$ & $239(93.7 \%)$ & \\
\hline No & $37(86.0 \%)$ & & NS \\
\hline Optimal oncologic therapy & $6(14.0 \%)$ & $3(1.2 \%)$ & \\
\hline Yes & & $252(98.8 \%)$ & \\
\hline No & $0(0 \%)$ & & NS \\
\hline Yes & $43(100 \%)$ & & \\
\hline
\end{tabular}

There are many reports indicating that advanced age is a predictor of poor prognosis in patients with endometrial cancer [1-8]. Although advancing age is associated with an increasing tendency toward high-grade endometrioid carcinoma, the histological characteristics do not appear to be able to entirely indicate the unfavorable prognosis of elderly patients with endometrial cancer $[2,6]$. Whether the poor prognosis among elderly patients can be accounted for entirely by a more advanced stage at the time of diagnosis, histology, or treatment or whether endometrial carcinoma among the elderly is intrinsically more aggressive than in younger patients remains controversial [2].

In the present study, histological type besides endometrioid adenocarcinoma $(\mathrm{p}=0.002)$ and histological grade 3 -endometrial cancer $(p=0.003)$ was more frequently diagnosed in elderly patients. Moreover, tumor with myometrial invasion depth $>1 / 2$ was also more frequently diagnosed in elderly patients $(\mathrm{p}=0.002)$. There were no significant differences between both groups in terms of clinical stages, lymph node metastases, or distant metastases. Benito $\mathrm{V}$ et al reported that endometrioid tumors were more frequently diagnosed in younger patients (78.8\% vs. $62.9 \%$ ), while type 2-endometrial cancer was more frequently diagnosed in elderly ones (37.1\% vs. $21.2 \%$ ) [6]. Other previous reports also showed that the biologic aggressiveness of endometrial cancer increases with age [2-5]. Our data were similar to data of these reports.

In our study, elderly patients received less pelvic lymphadenectomy $(\mathrm{p}=0.0004)$ than younger patients, while there were no significant differences between both groups in terms of hysterectomy, or para-aortic lymphadenectomy. In the Elderly patients, rates of optimal oncologic therapy according to the treatment principle of the institution was slightly lower compared to younger patients ( $86.0 \%$ vs. $93.7 \%)$, however the difference was not statistically significant. Benito $\mathrm{V}$ et al reported that elderly patients received less surgery (68.2\% vs. $92.4 \%)$, lymphadenectomy $(10.3 \%$ vs. $26.2 \%)$ and adjuvant treatment (37.1\% vs. $51.2 \%$ ) than younger patients [6]. In our institution, treatment for elderly patients with endometrial cancer seemed to be more aggressive than the institutions of previous reports.

In the present study, elderly patients had significantly frequent relapse of disease $(32.6 \%)$ more than the younger patients $(10.6 \%)(p=0.0005)$. Previous reports showed that advanced age is a predictor of poor prognosis in patients with endometrial cancer [2]. Our data was similar to data of these reports. In both elderly patients and younger patients, rates of optimal oncologic therapy according to the treatment principle of the institution was similar except a lymphadenectomy. Therefore, the reasons for poor prognosis of elderly patients include both high risk tumor biology and less aggressive operation including such as a lymphadenectomy.

\section{Conclusion}

The elderly patients with endometrial cancer had significant poorer prognosis than the younger patients, and reasons for this include both high risk tumor biology and less aggressive operation. 


\section{References}

1. Gayar OH, Robbins JR, Parikh K, Mei L, Thomas B, et al. (2011) Hysterectomy for uterine adenocarcinoma in the elderly: tumor characteristics, and long-term outcome. Gynecol Oncol 123(1): 71-75.

2. Poupon C, Bendifallah S, Ouldamer L, Canlorbe G, Raimond E, et al. (2017) Management and survival of elderly and very elderly patients with of endometrial cancer: An age-stratified study of 1228 women from the FRANCOGYN Group. Ann Surg Oncol 24(6): 1667-1676.

3. Giannice R, Susini T, Ferrandina G, Poerio A, Margariti PA, et al. (2001) Systematic pelvic and aortic lymphadenectomy in elderly gynecologic oncologic patients. Cancer 92(10): 2562-2568.

4. Lowery WJ, Gehrig PA, Ko E, Secorde AA, Chin J, et al. (2012) Surgical staging for endometrial cancer in the elderly - is there a role for lymphadenectomy? Gynecol Oncol 126(1): 12-15.
5. Rauh-Hain JA, Pepin KJ, Meyer LA, Clemmer JT, Lu KH, et al. (2015) Management for elderly women with advanced-stage, high-grade endometrial cancer. Obstet Gynecol 126(6): 1198-1206.

6. Benito V, Lubrano A, Andujar M, Mori M, Federico M, et al. (2019) Management of endometrial cancer in patients aged 80 years and older: Identifying patients who may benefit from a curative treatment. Eur J Obstet Gynecol Reprod Biol 242: 36-42.

7. Setiawan VW, Yang HP, Pike MC, McCann SE, Yu H, et al. (2013) Type I and II endometrial cancers: have they different risk factors? J cli Oncol 31(20): 2607-2618.

8. Bourgin C, Saidani M, Poupon C, Cauchois A, Foucher F, et al. (2016) Endometrial cancer in elderly women: which disease, which surgical management? A systematic review of the literature. Eur J Surg Oncol 42(2): 166-175.

\section{Your next submission with Juniper Publishers will reach you the below assets}

- Quality Editorial service

- Swift Peer Review

- Reprints availability

- E-prints Service

- Manuscript Podcast for convenient understanding

- Global attainment for your research

- Manuscript accessibility in different formats

( Pdf, E-pub, Full Tsext, Audio)

- Unceasing customer service

Track the below URL for one-step submission https://juniperpublishers.com/online-submission.php 